\title{
Correspondence
}

\section{Incorrect diagnosis of asbestosis}

SIR - In the paper 'The Epidemiology and Clinical Features of Asbestosis and Related Diseases' (Postgraduate Medical Journal, 1966, 42, 623-635), I referred to the difficulty of obtaining an estimate of exposure to asbestos and quoted the case of a woman diagnosed as asbestosis, whose exposure was non-industrial. This patient has since died and the autopsy reveals fibrosis but not asbestosis. I would like to report the case in more detail in order to emphasize the difficulty of making the diagnosis during life and to correct the false impression that the earlier report may have given.

\section{Case report}

Mrs T. G., born 1911. Worked in a fruit shop, a sweet factory and did domestic work before becoming a stitcher (mainly shirts). After getting married and raising a family she took in foster children but continued to work as a stitcher intermittently until she died.

During 1940-1 she helped her husband and another man build two wooden huts using asbestos sheeting for lining and partitions. The asbestos sheeting was hand sawn inside the huts. The patient and her husband lived in one hut for $2 \frac{1}{2}$ years before painting the inner (asbestos sheet) walls. They continued to use the hut as a week-end cottage for some years.

The husband (a seaman employed in the engine room) has similar chest $\mathrm{X}$-ray changes to the patient and is still alive but the other man died of carcinoma of the stomach with normal lung fields on X-ray in 1958.

The patient attended the Chest Clinic in 1957 because of an abnormal routine miniature chest film. She had a chronic cough dating back several years and recent breathlessness. Shadows were present in both lungs but as the mantoux and sputum cultures for tuberculosis were negative no antituberculosis treatment was given. She continued to attend the clinic with gradually increasing cough and breathlessness.

On 11 January 1965 she was admitted to Belfast City Hospital with left sided chest pain and severe breathlessness due to a spontaneous pneumothorax. No finger clubbing. Mantoux negative. Sputum negative for AFB on culture. ESR 28. Slight increase in $\alpha 2$ globulins otherwise biochemical and haematological investigations normal. The pneumothorax settled with conservative therapy but lung biospy was not considered justifiable because of it. The X-ray (reproduced in the original report) was interpreted as pulmonary fibrosis probably complicated by healed tuberculosis. By exclusion of other causes and because of the history of the asbestos huts, the fibrosis was thought to be asbestosis.

The patient attended the clinic with increasing symptoms and signs of wheezy bronchitis. ESR remained between 30 and $40 \mathrm{~mm} / \mathrm{hr}$. She complained of some difficulty swallowing. Barium studies of the oesophagus were normal but the trachea became deviated to the right. Bronchoscopy done at this time (May 1968) showed only marked rigidity of the trachea and main bronchi. Her condition continued to deteriorate gradually and when last seen ventilation tests were FEV 1.0 0.8 and FVC 1.3, improving to FEV 1.01 .0 and FVC 1.4, after bronchodilator aerosol. Contact was lost because of civil disturbances in the summer of 1971.

On 18 August 1972 she was admitted to Whiteabbey Hospital with fever, vomiting and a pain in the left chest. T. 100.6 ; P. 120 ; R. 26; B.P. $120 / 80$. Scattered rhonchi over both sides of chest more marked over the right upper lobe posteriorly. Hb. 12 g; W.B.C. 13,$815 ; 85 \%$ polys; ESR 16 . Sputum-no pathogens or AFB (repeated cultures for tubercle bacilli were also negative). Later she developed a yeast infection of the mouth and aspergillus was found in the sputum. Serological tests for Farmer's lung and aspergillosis $\underset{+}{\stackrel{\bar{\rho}}{+}}$ were negative.

She was treated with bronchodilators and a succession of antibiotics: ampicillin and cloxacillin, cephaloridine, cotri- $\frac{\bar{\sigma}}{\bar{\omega}}$ moxazole and then with amphotericin $B$ and caffeine iodide. $\frac{\Gamma}{\partial}$ She continued to run a fever of $100-102^{\circ} \mathrm{F}$, and died $\stackrel{\varnothing}{\varrho}$ suddenly of a massive haemoptysis on the twenty-third hospital day. No anti-tuberculosis therapy was given.

\section{Autopsy}

Death was due to haemorrhage into the lung and the only $\vec{\omega}$ relevant findings were in the lung.

Both lungs were attached to the chest wall by extensive? fibrous adhesions. There was a large multiloculated cavity in 3 the left upper lobe full of blood and surrounded by necrotic tissue. The rest of the upper lobe, and the apical and posterior basal segments of the lower lobe were firm and grey. The $i$ lingular and anterior segment of the lower lobe were of $\mathrm{N}$ relatively normal texture but contained aspirated blood.

The right lung showed a dense band of blackened fibrous $f$ tissue representing the posterior segment of the upper lobe and running down through the hilum to replace the middle lobe and part of the posterior basal segment of the lower lobe. The remaining right lung was relatively normal in $D$ texture but contained aspirated blood.

Histological examination confirmed the presence of extensive dense fibrosis and areas of caseation. The left and $\vec{v}$ some parts of the right lung showed active granulomatfs tissue with giant and epithelioid cell aggregates. Fungal elements were seen in some caseous areas but no acid fast bacilli and no asbestos bodies were seen in spite of a carefil search for both. The cultures taken at post mortem grew no tubercle bacilli. The pathologists diagnosed chronic active fibrocaseous tuberculosis.

\section{Differential diagnosis}

In spite of the histological findings it seems unlikely that this was a simple case of tuberculosis with cavitation and terminal haemorrhage. The main reasons for doubting this diagnosis are the negative mantoux tests and negative sputum examinations and cultures for tubercle bacilli over a 15-year $\bar{\partial}$ period of observation and after death in spite of no anti- 3 tuberculosis treatment being given.

The radiological changes in the lungs over the years were unlike tuberculosis. In 1957 there were bilateral lower zone linear opacities. By 1962 the changes involved all zones of $\delta$ both lungs with small irregular opacities superimposed on the linear ones. The lesions were more numerous peripherally and 0 the clear outline of the diaphragms was lost. By 1965 at the time of the pneumothorax there were larger irregular $D$ opacities especially in the right lower and left upper zone with distortion of the lung pattern. From 1965 until 1971 the $X$-ray changes showed coalescence and hardening of the $O$ linear and irregular shadows with progressive distortion of $\mathrm{N}$ the normal architecture. There was contraction of the upper lobes with pulling up of the hilar and displacement of the $\mathrm{\omega}$ trachea to the right, the diaphragms were raised and tented. 0 The lateral films showed the development of the dense scar in the region of the oblique fissure on the right side. A film taken during the terminal illness showed further progression $\mathbb{D}$

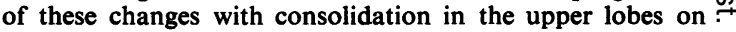
both sides.

The X-ray changes suggested a widespread pulmonary $\vec{O}$ disease leaving coarse scarring, more like progressive massive $\overrightarrow{\mathbb{D}}$ fibrosis than tuberculosis. They were compatible with an $\frac{\rho}{\mathbb{D}}$ inhaled dust disease complicated by either an immune $\stackrel{\mathbb{Q}}{\varrho}$ response (such as Farmer's lung disease) or by an autoimmune 
response such as the interaction between silicosis and rheumatoid disease. The diagnosis of asbestosis complicating inactive tuberculosis that was made in 1965 became less likely with the progression of the lung lesions.

However, the absence of significant dust in the lung at post mortem and the patchy distribution of the lesions with apparently normal lung between made it very unlikely that any inhaled dust contributed to the disease. No serological evidence of immune or autoimmune disease was obtained during life.

The presence of caseation is usually taken to rule out the diagnosis of sarcoidosis and the absence of sarcoid lesions in other organs makes this diagnosis unlikely. One must assume that the fungal elements in the lungs and sputum were a terminal complication.
This case illustrates the difficulty of diagnosing some cases of progressive lung disease even after death. Lung biopsy might have excluded asbestosis in this case but was considered too hazardous because of the extensive scarring and previous pneumothorax. Since 1965 I have only seen clinical asbestosis after relatively prolonged occupational exposure in Belfast. The only serious complication of non-industrial exposure observed here so far is mesothelioma.

P. C. Elmes. M.D., F.R.C.P. Whitla Professor of Therapeutics and Pharmacology, The Queen's University of Belfast, Institute of Clinical Science,

Grosvenor Road, Belfast, BT12 6BJ. 